\title{
Charm on Modern Art Design Based on the Fusion of Light and Shadow
}

\author{
Xiaohua Ge \\ Huanghe Science and Technology College \\ Zhengzhou, China 450063
}

\begin{abstract}
As one of indispensable design elements, optic artistry makes the expressions of object images more vivid when used in modern art design. This paper analyses the significance and various forms of lighting techniques from four aspects including the fusion of lighting techniques and modern art design, realization forms of lighting techniques in the modern art design, the appliance of lighting techniques in the modern art, and the charm and value of lighting techniques in the modern art works, in order to comprehensively illustrate the necessity of the fusion of light and shadow in modern art design.
\end{abstract}

Keywords - light and shadow; fusion environment; modern art design; charm and value

\section{INTRODUCTION}

With the development of the times, people's aesthetic appreciations and pursuits of modern art are enhanced gradually, and in the meanwhile, the means and methods of artistic creations also diversify. The works of modern style integrating means of expressions with various art elements such as the integration of painting, music, literature and art as well as traditional arts have been common to us all. The fusion of a variety of elements not only makes people enjoyable, but promotes the development of modern art. In the joint development of various arts, the neglected optic artistry around us is used in another way by smart designers. Therefore, optic artistry then becomes the product of the times.

\section{ORGANIC INTEGRATION OF LIGHT AND SHADE AND MODERN ART DESIGN}

Art shares much in common. In the development of modern art such as paintings, design, photography, architecture and other aspects, there also exist art, physique and image perception of space as well as virtual-real changes. That is why the effect of the relationship of light and shade in the actual situation very important. As a kind of art, optic artistry itself exists in modern art design in the form of performance element It is the organic integration of static and dynamic just like a jumping fairy of performance techniques in modern art design.

It is obvious that the light reflection is very important in modern art design. With the effect of light and shadow, works can produce different colors as well as light and shades. But what works to the brilliant works of art is how to achieve the organic performance of light and shade. In the creation of paintings, photography, shadow figures and design, elements are only elements, and the essence of works is how to use. In performance techniques, light reflection is actually the most simple, effective and inexpensive. In modern art design, we can create shadows and patchy shadows through natural light, form different forms or patterns by the using of intensity and direction of shadow, create the atmosphere of works by using the shadows of light to highlight the smart feelings of works; we also can use light reflection to suggest stories, or show our design attitudes and so on. Audience's feelings transcending appreciation values of the art works can be received by using different ideas and methods of expressions. The works presented are not just the simple objects of surface value, but people's views and thoughts on observing the world. Although the design of the works shows basic composition rules, it will help people to create their own styles on the condition that they can control them.

Not merely a means of art, light reflection is the product of art and fashion as well as an expression of the soul of works. Besides, it has infinite expressions and variants. In contemporary society, art is established based on natural and growing culture and objects. Integration of light reflection and art is the developing trend of times: currently, a variety of virtual techniques to catch the reality of feeling such as 3D, 4D, $5 \mathrm{D}$, AR, three-dimensional projection lights depend on the integration of light reflection and modern art design. They are dreamlike, full of endless inspiration. Generally, visual art cannot do without light and shadow. In fact, many modern arts are the products of human vision through intermedium.

\section{THE FORMAL EXPRESSION OF LIGHT AND SHADOW IN THE MODERN ART DESIGN}

Light is indispensable in life, and shadows can be formed with it. In earlier times, people use light to meet their basic living needs. Light and shadow are indispensable natural things in human life. In the process of growing satisfaction of basic living, human begin to pursue spiritual life; driven by the social formation, light reflection is applied to all aspects of life as a new technology. Naturally, the combination of lighting techniques with the modern art design also come into being, and it produces endless charm effects by using the unique relationship between objects and light. In modern art design, light reflection produces different artistic effects for 2D and $3 \mathrm{D}$ of static and dynamic mainly through light sources, and the effects are more obvious especially in the aspects of music fountain and stage design. 
In the process of artistic expression of modern art design, the application and the form expression of light and shadow are fully expressed in modern design ideas, thinking and artistic practices through the designers' in-depth understanding, analysis, recognition and using light reflection as a form of expression.

\section{A. The Lighting Reflection Performance of Stereo and Visual Impact in Modern Art Design}

In modern design, artistic forms are very important in design. In the process of applying lighting, the features of naturalness, directions, permeability and brightness are often used to create the object shapes or outlines, textures and beauty of materials to achieve stereo and visual impact.

\section{B. The Formal Expression of the Space-time Beauty of Lighting Reflection in Modern Art Design}

In modern design, people often create changing modern design works with the features of perceptibilities, strong comparation, shrinkage and tensile and self-development according to the specialities of natural lighting including seasonal variation, different time and moments, naturalness of all things in the world to enhance ornamental and artistic connotation of works and achieve the effect of space-time beauty.

\section{Formal Expressions of the Space of Light and Shadow in Modern Art Design}

In modern design, it is very important to create spaciousness. In the application of light, people often create works of art with crossing effects such as space, deficiency and excess, loose and draw, thick and thin, interruption and continuity, and transition by using their features of space shaping force, streamline guiding force, and space force series to deepen the sense of space, cohesion and organization of work space with the aim to achieve representation effects of spaciousness.

\section{APPlication of Light ANd Shade IN Modern ART DESIGN}

With the continuous development of science and technology as well as the times, the design of modern art is constantly improving and innovating. For graphic design, light reflection is only the application of "lighting"; however, for space design, it is one of the important factors to achieve threedimensional modeling. With the innovation of technology, the fusion of light and shade not only works as a kind of specific language surpassing culture, territory, nations, and states, but also is used widely in art based on the modeling languages of different colors, shapes, materials.

Driven by natural light, light and shadow present diverse forms from basic lighting to different lighting effects in later period, in which the most prominent is the design depth and space effect that modern designers have reached by using light and shadow: they use the effects of light and shadow of light projection, hard and dim light, color changes, light shapes, light formations, and gradual changes to produce a variety of vivid sensory effects. With the development of information age, the application of light in the modern art design is not only based on the simple manifestation of the sense of space and sense of modeling, but plays more humane and higher quality on the road of modern art design. Besides, it will combine with a variety of information technology including multimedia, nature, music and other means to achieve mutual resonance and show its colorful art of the times. Designers can express design works more artistically by using lighting reflection to show the works of art and enhance the aesthetic value of the works. Designers can experience coming science and virtual scenes through light reflection to achieve new effects of the art works. In order to achieve the design purpose, designers usually use the light reflection as their own "actor" through rich artistic creativity and imagination to play the role of light to its utmost in modern art design. What's more, more designers seize the unique characteristics of light such as reflection, back light, front light, light sources, and use the unique means of new technology, color, medium and so on to apply lighting techniques to design works incisively and vividly with the aim to make the works more prominent and achieve the maximum effects. The application not only reflects the value of light and shadow in modern art design, but also reflects the progress of society, culture as well as science and technology.

With the development of society, lighting techniques only works as an expressing method of visual expression used in modern design, and the expression effects of light and shade still need to rely on modern designers' understanding. Therefore, there exist some defects in the application of lighting techniques, which require us to find new breakthroughs and applications in the lighting techniques in the development of society, times, science and technology as well as culture.

\section{THE Charm AND VALUE OF Light AND SHADOW IN MODERN ART WORKS}

In modern art, the charm and value of light and shadow lie in that it endows works with different tastes such as sketches, oil paintings, and gouaches in traditional paintings. Illuminated by light, the works show different light and shade, colors, and contrast making great difference in artists' works. Having strong artistic attractions, light reflection can produce a variety of changes and affect creative ideas, techniques and expressions of artists. Combined with light and shade, art works can reach the effect of creating an atmosphere and shaping the shape and so on to show the proportion of light and shade to help artists create different works.

In addition, light and shade can also be used as a part of works presented to the audience. The artistic effects depend on whether artists can use the works to express their visual beauty and inspire the audience to have the same aesthetic feelings. In modern art, artists' excavation, fantastic conception and design as well as their creation of abstract, refined, typical visual effects are the key to achieve good effects of light and shadow. Light reflection is an important means of the expressions of modern art works, and higher artistic state can be reached by using it. Light reflection not only diversifies languages in modern art, but also enhances the aesthetic connotations and characteristics of modern art, and in the meanwhile, it 
enhances the expressive forces of art works and gives a greater impact on works. According to their own creative needs, artists can use the charm and value of light and shadow to design deep, amazing, and more delicate artistic works in modern art.

Modern artists create the atmospheres of art works through light reflection such as the cases that modern artists create stereoscopic impressions of objects through the full use of light and shade. By exploring the principles of the light reflection, artists can create 3D objects and 3D world through light and shadow and achieve the visual effects under the light to feel amazing art works. In modern art works, many factors have influences on the atmosphere and the most important one is light reflection. Light reflection can not only create shadow and three-dimensional shapes, but create a different picture by using same brightness, colors and other factors. Working as an important painting language, light reflection plays an important role in truly 3D visual effect and practical aspects. What's more important, it can enhance the art atmosphere, which truly reflects the artists' subjective feelings and art expressions. Besides, light reflection can also be used to express modern artists' true feelings, such as expressing feelings according to the levels of brightness. Bright and warm light endows people with calm and happy feeling, while dim and hazy light brings depression and melancholy to people. Artists can express whatever they want by using light.

\section{CONCLUSION}

In modern art design, many works are integrated with light and shade. In this sense, the light can be called the soul of modern art design, and developing the application of light and shadow has become an important task of modern art design; in the meanwhile, modern art design in the fusion of light and shadow has developed into an important research field. Different effects of light convey different artistic effect. Different light and shade create different visual perceptions, and it is the embodiment of the latest achievements in science and technology that light and shade effectively combinate with the design of modern art. Light, shadow, sound and other factors should be skillfully integrated in order to expand the scope and breadth of light and shade, which requires designers to reserve abundant knowledge and accumulate practical experience, strive to explore, and try out using different light and shade constantly to effectively promote the sustainable development of modern art design.

\section{REFERENCES}

[1] He Shufang. The Poet of Light-An Analysis on Rembrandt's Processing of Light and Color [D]. Academy of Fine Arts, Xuchang University, 2009

[2] Li Beilei. The History of Western Art [M]. Chongqing: Chongqing University Press, 2010

[3] Yang Zheng. The Expression of Light Reflection and Art [J]. Journal of Taizhou Polytechnic Institute, 2007, 7 (6):31.

[4] Zeng Yan. The Study of the Appliance of Light in Jiangnan Private Space [J]. Guangdong Landscape, 2008.30 (3):17

[5] Ma Jianzhong, Suo Xiaoling. The Picture Appreciation of Animation [M]. Beijing: Communication University of China Press, 2011

[6] Han Qing. The Visual Performance of Light and Shade in the Show Design, Master's Thesis of Shanghai Normal University, May 2010
[7] Lv Lina, Zhuang Yu. Study on Virtual and Gathering Space from the Digital Image Art, "Science and Technology Information" [J], 2010 Seventh

[8] Xu Aiguo. An Analysis on the Role of Light in Stage Performances [J]. Friend of Science Amateurs, 2012 (6).

[9] Zheng Huilin. An Analysis on the Role of Art Effect of Stage Lighting on Stage Performance [J]. Social Science, 2012 